\title{
Computational Fluid Dynamics and Its Applications 2012
}

\author{
Guan Heng Yeoh, ${ }^{1,2}$ Chaoqun Liu, ${ }^{3}$ Jiyuan Tu, ${ }^{4}$ and Victoria Timchenko ${ }^{2}$ \\ ${ }^{1}$ Australian Nuclear Science Technology Organisation (ANSTO), Locked Bag 2001, Kirrawee, NSW 2233, Australia \\ ${ }^{2}$ School of Mechanical and Manufacturing Engineering, University of New South Wales, Sydney, NSW 2052, Australia \\ ${ }^{3}$ Centre for Numerical Simulation and Modeling, Department of Mathematics, University of Texas at Arlington, Arlington, \\ TX 76019-0408, USA \\ ${ }^{4}$ School of Aerospace, Mechanical and Manufacturing Engineering, RMIT University, P.O. Box 71, Bundoora, VIC 3083, Australia
}

Correspondence should be addressed to Guan Heng Yeoh, guan.yeoh@ansto.gov.au

Received 9 September 2012; Accepted 9 September 2012

Copyright ( 2012 Guan Heng Yeoh et al. This is an open access article distributed under the Creative Commons Attribution License, which permits unrestricted use, distribution, and reproduction in any medium, provided the original work is properly cited.

The launch of this annual focus/special issue in this year has been built upon the success of the special issue that was introduced last year in the published special issue of Modeling and Simulation in Engineering. There is no doubt that there is an increasing resurgence of computer simulation technology changing or reshaping the landscape on how computational fluid dynamics, better known by its acronym CFD, is currently being applied in many fields of engineering research and application. For this particular issue, we have managed to collate a range of topics and papers that pertinently encapsulate the many advances and applications of CFD which we have the great pleasure of sharing with the wider community. We certainly would like to thank the numerous authors for their excellent contributions and patience in assisting us. More importantly, we would like to warmly acknowledge the tiresome work of all referees in reviewing these papers.

This annual focus/special issue contains eleven papers. The advances and wide applications of CFD in each of the papers are detailed in the following.

"New theories on boundary layer transition and turbulence formation" by C. Liu et al. presents a short review on the investigation of late boundary layer transition and turbulence via direct numerical simulation (DNS). A new theory has been proposed which comprises receptivity, linear instability, large vortex structure formation, small-lengthscale generation, loss of symmetry, and randomization to turbulence. A new theory on turbulence is also proposed which comprises all small length scales generated by shear linear instability rather than vortex breakdown through multiple-level ejections and sweeps and consequent multiple-level positive and negative spikes. Through their DNS observation, energy is transferred from large to small vortices by multiple-level sweeps which are in contrast to Kolmogorov's theory where the cascading of energy occurs from large to small vortices through vortex stretch and breakdown.

"The role of mesh generation, adaptation, and refinement on the computation of flows featuring strong shocks" by A. Bonfiglioli et al. presents some recent advances in computational approaches to better capture shock waves. There is a strong relationship between the development and application of mesh adaption/refinement/generation techniques and the computation of high-quality solutions of flows featuring strong shocks. Mesh adaption/refinement/generation techniques that can adapt to the shock front have been found to be a key ingredient in achieving accurate solutions for this kind of flow fields. Nevertheless, more relevant and conclusive enhancements in terms of solution quality can be obtained by means of a shock-fitting technique for unstructured grids. Indeed, the use of this newly developed technique completely removes the capturing process which has been the source of most of the problems that have plagued current shock-capturing schemes.

"Landing gear aerodynamic noise prediction using building-cube method" by D. Sasaki et al. presents the application of a building-cube method to solve the fluid flow around a JAXA landing gear. The building-cube method is a multiblock-structured Cartesian mesh solver. Here, the computational domain can be decomposed of assembly 
of various sizes of building blocks where smaller blocks are employed to capture the fine details of the fluid flow. The airframe noise is predicted through the coupling of incompressible Navier-Stokes flow solver and the aeroacoustic analogy-based Curle's equation to predict the acoustic sound generated from the object in the fluid flow. Good agreement is achieved between the experimental data and numerical predictions. The results also show that the torque link position highly affects the flow acceleration at the axle region between two wheels, which causes the change in sound pressure level at the observation point.

"Turbulent and transitional modeling of drag on oceanographic measurement devices" by J. P. Abraham et al. presents the use of CFD in determining the drag on oceanographic devices (bathythermographs). Such devices are employed to monitor changes in ocean heat content. Fall-rate equation is reasonably accurate for ocean environments that match the experiments from the correlations developed. In order to better improve drag calculations which can significantly impact the estimation of ocean heating associated with global warming, a CFD approach has been undertaken to provide drag coefficients which can be used to predict depths independent of a fall-rate equation.

"A Study of the location of the entrance of a fishway in a regulated river with $C F D$ and $A D C P$ " by A. G. Andersson et al. presents the application of CFD to determine the flow downstream of a hydropower plant with regards to upstream migrating fish. Field measurements via an Acoustic Doppler Current Profiler (ADCP) were utilised to purposefully validate the CFD simulations. Various configurations were investigated in order to assess the flows at the fishway entrance and further downstream, where the flow from the turbine meets the old river bed, which represent the current fish passage for upstream migrating fish.

"Numerical model for cavitational flow in hydraulic poppet valves" by S. I. Bernad and R. Susan-Resiga presents the CFD investigation of two-phase flow inside a poppet valve. A mixture model is applied to predict pressure and density distributions of the flow inside the cavity. Cavitation model that utilizes the modified Rayleigh-Plesset equations for bubble dynamics is adopted. The cavitating flow pressure distribution displays a constant value inside the vapour-filled cavity, with a slow increase as the mixture density increases. The investigation of the liquid-gas mixture density shows a smooth and continuous transition from vapour to liquid. This transition takes place over a relatively large region.

"Simulating the effects of structural parameters on the hydraulic performances of venturi tube" by Y. Sun and W. Niu presents a parametric CFD investigation to assess the effects of structural parameters (such as throat taper, throat contraction ratio, and throat length) on the hydraulic performance (such as outlet faceted average velocity, minimum pressure, and critical pressure) of a Venturi tube under different inlet pressures and pressure differences between inlet and outlet. The throat contraction ratio is found to be the contributing factor to the Venturi injector performance.

"Computational fluid dynamics modelling and experimental study on a single silica gel type B" by J. White presents the application of CFD in in the area of porous media and adsorption cooling system. CFD can be a useful tool to predict the water vapour flow pattern, temperature, heat transfer, flow velocity and adsorption rate. By obtaining useful experimental data from thermogravimetric measurements, CFD predictions are found to be comparable with the measured data. This implies that CFD could provide useful information for the design of adsorption cooling systems and better predict the system performance.

"A CFD simulation on how the different sizes of silica gel will affect the adsorption performance of silica gel" by J. White presents a review on the application of CFD in simulating the adsorption of water vapour on silica gel granules and to study the effect of granule sizes indicating that reducing the granule size increases the adsorption. CFD has been found to be a promising tool in evaluating the heat transfer behaviour in an adsorption bed. Further CFD studies are required for evaluating the adsorption performance with a larger silica gel and porous media.

"Determination of flow conditions in coronary bifurcation lesions in the context of the medina classification" by M. M. Zarandi et al. presents the use of CFD in understanding the flow condition and wall shear stress distributions in various bifurcation lesion types in a coronary artery bifurcation. The flow ratio and time-dependence patterns of WSS resulting from the numerical simulation of pulsating hemodynamic flows are investigated in healthy and stenosed coronary artery bifurcations. Various stenoses configurations are considered in the context of Medina lesion classification, and a detailed numerical result for time-dependent wall shear stress distributions that may be involved in lesion initiation and progression is presented. The results indicated that in the bifurcation lesion types, the flow condition and wall shear stress distribution in side branch are influenced by the lesion morphologies which cannot be fully assessed by quantitative coronary angiographic parameters.

"Detailed CFD modelling of open refrigerated display cabinets" by P. D. Gaspar et al. presents a comprehensive CFD modelling of air flow and heat transfer in an open refrigerated display cabinet (ORDC). The physicalmathematical model considers the flow through the internal ducts, across fans and evaporator, and includes the thermal response of food products. Air humidity effect and thermal radiation heat transfer between surfaces are accounted in the model. Experimental tests are performed to characterize the phenomena near physical extremities and to validate the numerical predictions. The agreement between numerical predictions and experimental results is found to be satisfactory and adequate for these types of engineering problems. Such predictive capabilities of the computational model could be used to promote a range of geometrical and functional parametric studies that could better improve the thermal performance of the ORDC and consequently food safety.

Guan Heng Yeoh Chaoqun Liu Jiyuan Tu

Victoria Timchenko 

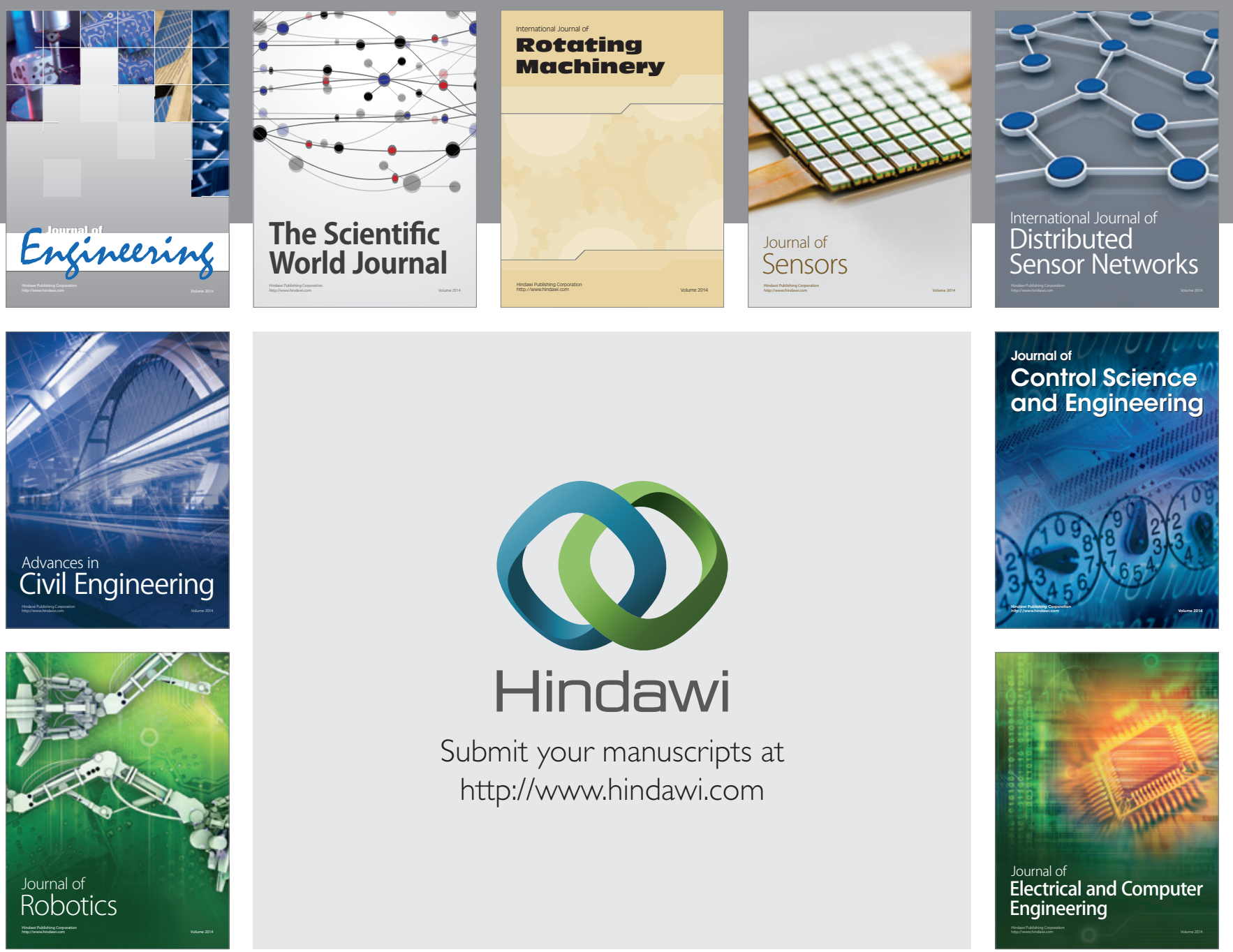

Submit your manuscripts at

http://www.hindawi.com
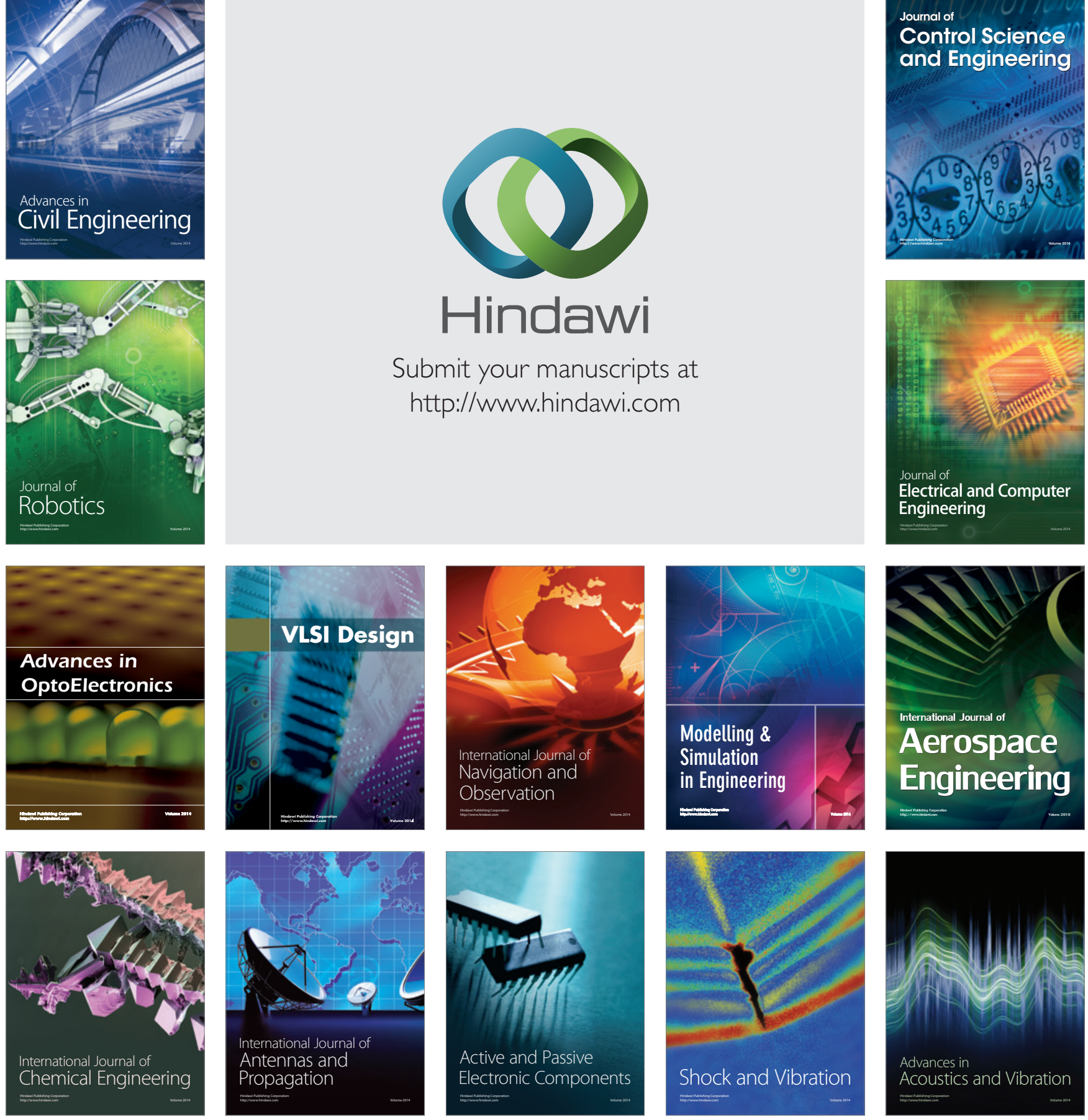